\title{
Estudio de los criterios para la selección de centros de prácticas de calidad. Una propuesta de evaluación para mejorar la formación de los pedagogos
}

\author{
Study of the criteria for selecting quality schools for teaching practice. \\ An assessment proposal for improving teacher training
}

\author{
Sara Serrate González, ${ }^{a}$ Sonia Casillas Martín, ${ }^{a b}$ Marcos Cabezas González ${ }^{a c}$ \\ ${ }^{a}$ Universidad de Salamanca \\ Telf.: (34) 9232946 30. Correo electrónico: sarasg@usal.es \\ b Telf.: (34) 9232946 30. Correo electrónico: scasillasma@usal.es \\ c Telf.: (34) 9232946 30. Correo electrónico: mcabezasgo@usal.es
}

\begin{abstract}
RESUMEN
El presente artículo recoge los resultados de un estudio sobre las prácticas externas del Grado en Pedagogía, realizado con el objetivo de aportar criterios de calidad para la selección de centros adecuados al perfil profesional del pedagogo y hacer recomendaciones sobre la oferta formativa práctica. Para la realización del estudio, de enfoque metodológico cualitativo, se diseñó un cuestionario de respuestas abiertas con la finalidad de que los propios alumnos valorasen la idoneidad de los centros en donde habían realizado las prácticas. La muestra quedó conformada por 58 alumnos y 48 centros colaboradores con las prácticas externas. Para valorar las respuestas, se ha realizado un análisis de contenido para extraer los factores y relaciones más intensas. A partir de los resultados obtenidos, se han podido concretar, de forma minuciosa, los elementos claves y útiles para la selección de centros idóneos para la realización del Prácticum.
\end{abstract}

Palabras clave: prácticas, formación profesional, criterios de calidad, evaluación.

\begin{abstract}
This article presents the results of a study on the external practice teaching, linked to the undergraduate degree in education. Our aim was to contribute criteria on quality in the selection of schools to fit the professional profile of the student teacher and to make recommendations in relation to the schools available for teaching practice. To carry out the study, which employs a qualitative methodology, an open questionnaire was drawn up so that the students themselves could appraise the suitability of the schools where they had done their teaching practice. The final sample comprised 58 students and 48 schools that collaborate with teaching practice. Content analysis was used to evaluate the responses to extract the factors and the most intense relations. Based on these results we have been able to specify, in great detail, the useful key elements for selecting the most suitable schools for teaching practice.
\end{abstract}

Key words: practices, training, quality criteria, evaluation. 
Estudios Pedagógicos XLII, N 3: 369-389, 2016

ESTUDIO DE LOS CRITERIOS PARA LA SELECCIÓN DE CENTROS DE PRÁCTICAS DE CALIDAD. UNA PROPUESTA DE EVALUACIÓN PARA MEJORAR LA FORMACIÓN DE LOS PEDAGOGOS

\section{INTRODUCCIÓN}

La puesta en marcha de unas buenas prácticas para los futuros pedagogos depende, en gran medida, de la calidad de los centros donde se van a desempeñar las mismas. La realización de investigaciones en este sentido es fundamental para aportar datos que favorezcan la mejora en este ámbito de estudio tan importante: las prácticas profesionales.

Este artículo presenta un estudio sobre las prácticas externas del Grado en Pedagogía realizado con el objetivo de aportar criterios para la selección de centros adecuados al perfil profesional del futuro pedagogo y hacer recomendaciones sobre la oferta formativa práctica que puedan suponer una mejora para el conjunto del sistema universitario.

La materia Prácticum es una asignatura formativa que potencia los conocimientos, habilidades y actitudes del alumno, y que comporta la aplicación de propuestas educativas y socioeducativas en contextos profesionales reales específicos. Es por ello que las prácticas externas suponen una "vía para superar la posible fragmentación del conocimiento adquirido a lo largo de las diferentes disciplinas" (Bas, 2005, p. 192) a través de una estrecha relación y articulación entre la teoría y la práctica profesional, que permite al alumnado la posibilidad de "construir un pensamiento práctico" (Morales, 2013, p. 350) a través del desarrollo de instrumentos, recursos y formación específica que permiten ir formando una identidad profesional propia, necesaria para su posterior inserción en el mundo laboral (López e Hinojosa, 2008).

En la misma línea, Valle y Manso (2011) reconocen que las prácticas constituyen uno de los principales pilares de la formación inicial, pues se trata del primer contacto con la realidad de su futura profesión, permitiendo desempeñar su labor en un ámbito similar al que en el futuro se encontrarán, adquiriendo, además, distintas actitudes, aptitudes, destrezas y competencias profesionales.

El Prácticum es un elemento esencial para la formación práctica universitaria con implicación en el buen hacer de la profesión. Las investigaciones ponen de manifiesto cómo en los primeros años de práctica profesional se definen y marcan el estilo, los valores y la deontología profesional (Bolívar y Domingo, 2006; Flores, 2006; Marcelo, 2009). Asimismo, "será en el encuentro entre la teoría y la práctica donde podrá construir su propio conocimiento, aprender a actuar, asumir actitudes profesionales" (González, 2009, p. 36), puesto que supone un acercamiento y complemento del aprendizaje académico, más teórico, a la realidad profesional de cada titulación (González y Hevia, 2011). Proporciona al alumnado un espacio y tiempo formativo para aprender a partir de la observación, reflexión y acción en un contexto determinado, facilitando la comprensión de las peculiaridades propias de cada titulación.

No obstante, la importancia del Prácticum no radica únicamente en las posibilidades formativas que ofrece al alumno en el desarrollo de sus competencias, sino que, en tanto espacio profesionalizador, supone una oportunidad para redefinir los ámbitos profesionales de cada titulación (Zabalza, 2006).

Parece evidente que esta asignatura está compuesta por múltiples variables, constituyendo una realidad compleja en la que participan tres agentes fundamentales: los estudiantes, la institución universitaria y los centros de prácticas (Zabalza, 2011). Uno de los agentes de mayor peso dentro de este proceso de formación práctica es el centro o institución donde se van a realizar. Estudios recientes como los realizados por Zabalza (2011) y Armengol et al. (2011) ponen de manifiesto que es necesario revisar de forma exhaustiva la formación que proporcionan los centros e instituciones profesionales que acogen al alumnado, en un 
intento de mantener una interrelación positiva que ayude a configurar una asignatura más adaptada y específica a las demandas y necesidades educativas y sociales actuales.

Como consecuencia, el periodo de formación en los centros sería conveniente ejecutarlo en instituciones educativas seleccionadas o reconocidas por su excelencia profesional, por sus programas pedagógicos, por su forma de llevarlos a cabo y también por los resultados obtenidos a nivel social, más allá de los puramente académicos. Con estos antecedentes, las prácticas de la formación inicial puede que no lleguen a tener la calidad deseada si no se seleccionan los centros más adecuados a la titulación correspondiente (Valle y Manso, 2011).

En el caso del Grado en Pedagogía de la Universidad de Salamanca, las prácticas se desarrollan durante el cuarto curso y tienen una carga de 24 créditos ECTS. Se contemplan dos menciones para la realización de las prácticas: la de Formación y Gestión de calidad y la de Orientación Educativa y Asesoramiento. Hay que tener en cuenta que un amplio número de alumnos elige la opción "sin mención" para la realización de las prácticas, contemplada también en el plan de estudios, y los centros asignados para realizar las prácticas de estos estudiantes suelen ser instituciones de ámbito social, elegidas normalmente por el propio alumnado.

Desde la Universidad se cuenta con una estructura organizativa de coordinación del Prácticum que, aunque dispone de una amplia autonomía institucional (Gairín y Martín, 2004), debe asumir, en un acto de responsabilidad, un conjunto de tareas sobre la gestión de las prácticas externas. El abanico de funciones a desempeñar para el buen funcionamiento del Prácticum abarca desde la gestión de la información al alumno, la canalización de la oferta de plazas, hasta la adjudicación de las mismas.

Se establece un contacto previo con algunos centros por medio de seminarios en la Universidad impartidos por los responsables de diversas instituciones profesionales colaboradoras con las prácticas universitarias. Con estos encuentros, se pretende dar a conocer las distintas instituciones en las que pueden desempeñar un trabajo profesional los pedagogos, además de ofrecer al alumnado una visión amplia de los diversos ámbitos de intervención profesional relacionados con su profesión y que descubra aspectos esenciales, principalmente teóricos-prácticos, del trabajo que el pedagogo realiza en cada espacio profesional (Morales, 2010).

Una vez impartidos estos seminarios formativos, se procede a la elección y distribución de los centros entre el alumnado, considerado un aspecto esencial dentro del proceso formativo. Se les ofrece la posibilidad de buscar y gestionar plaza en aquellos centros en los que les gustaría realizar sus prácticas y con los que la Universidad no tiene concertadas plazas para el Prácticum, y a los que podrán incorporarse una vez aceptados y firmado el convenio de cooperación correspondiente. Posteriormente, desde la Universidad se realiza una estimación de oferta de plazas y del alumnado matriculado en cada curso académico. Para aquellos estudiantes que no hayan gestionado su plaza previamente, se realiza una difusión de la información sobre las entidades colaboradoras de las prácticas con la Universidad, y se presenta una oferta de plazas disponibles en aquellos centros que tienen convenio con la Facultad, ofreciéndoles un abanico amplio de instituciones donde pueden hacer sus prácticas externas, de tres ámbitos fundamentalmente: orientación educativa, social y de formación y gestión.

El procedimiento de adjudicación de plaza se realiza teniendo en cuenta criterios de transparencia, asignando los centros de acuerdo al orden de preferencia que indique el alumno. Con una oferta de plazas superior a los estudiantes potenciales del Prácticum, entre la variedad de centros e instituciones ofertadas se encuentran: 
a. Centros de formación y gestión: Centros de Planificación y Gestión Educativa, Programas propios de la Universidad de Salamanca, Programas promovidos por Diputaciones y Ayuntamientos.

b. Departamentos de Orientación de Institutos de Enseñanza Secundaria y Centros de Infantil y Primaria. Gabinetes y equipos psicopedagógicos en instituciones y entidades públicas y privadas.

c. Centros especializados en intervención social con personas con discapacidad, drogodependientes, inmigrantes, tercera edad, en protección y promoción de la mujer, desarrollo comunitario, en menores y jóvenes, prevención de la delincuencia, ocio y tiempo libre, centros hospitalarios, etc.

Sin embargo, se encuentran algunos factores institucionales o condiciones contextuales a la hora de realizar un adecuado aprovechamiento del periodo de formación práctica por parte del alumno, tales como la formación y el perfil del tutor profesional de las prácticas, las tareas y actividades diseñadas para que el estudiante realice en el centro de prácticas, las posibilidades de inserción laboral, la disponibilidad de centros para cada curso académico, etc.; aspectos, todos ellos, que van a influir en la evaluación de la idoneidad de las diferentes instituciones para recibir estudiantes en prácticas (Casillas, González y Serrate, 2015). Sin simplificar el peso que tienen dichos factores, consideramos que disponer de herramientas de evaluación de los diferentes aspectos implicados en la selección de instituciones en prácticas puede contribuir a mejorar el proceso de aprendizaje práctico en la Universidad (Valle y Manso, 2011). Una vez adjudicados los centros de prácticas, el alumno se encuentra inserto académica y profesionalmente en los mismos durante cuatro meses; durante ese tiempo se mantienen reuniones semanales entre el alumno, el tutor profesional y el tutor académico. No obstante, debemos tener en cuenta que es el alumno quien convive durante este tiempo con el tutor y el equipo de profesionales en la institución de destino, $\mathrm{y}$, por ello, son los mismos estudiantes quienes pueden relatar de una forma coherente si se cumplen o no los objetivos marcados en un proyecto formativo que se firma al inicio de este periodo formativo. En este sentido, a partir de los relatos de los estudiantes que valoran la adecuación de los criterios de selección de instituciones que ofrezcan unas adecuadas y beneficiosas prácticas externas, se consigue una mejor discriminación de centros que ofrezcan prácticas educativas de calidad.

Por las razones expuestas, el equipo responsable de las prácticas externas de la Facultad de Educación de la Universidad de Salamanca ha trabajado en la elaboración de un instrumento para la valoración y clasificación de los centros de prácticas, que nos permite seleccionar aquellos centros que garanticen unas prácticas de calidad, así como jerarquizarlos en función de sus aportaciones formativas a la profesión del pedagogo.

Las conclusiones de este trabajo resultarán de gran aplicabilidad para facilitar el arduo trabajo que deben realizar las Universidades en la formación inicial práctica de su alumnado y las propias instituciones educativas en lo relativo a las prácticas externas.

\section{DISEÑO DE LA INVESTIGACIÓN}

\subsection{OBJETIVOS}

Este trabajo pretende responder a la preocupación existente sobre la selección de centros para la realización de buenas prácticas educativas para los futuros pedagogos y 
buscar una herramienta que ayude a las universidades a distinguir cuáles son los centros de práctica más óptimos para formar al alumnado. No supone un objetivo para nuestro estudio comprobar ni valorar el funcionamiento interno de los centros, puesto que se trata de instituciones reconocidas social y educativamente, por lo tanto, se da por supuesto que cumplen una labor excelente hacia los usuarios y la ciudadanía en general. Esto no quiere decir que los recursos personales, espaciales y temporales disponibles posibiliten que determinados centros sean más adecuados que otros para colaborar con la formación inicial práctica de los universitarios.

Como objetivo general se pretende conocer la percepción del alumnado sobre el centro o institución en el que ha ejecutado las prácticas externas de su titulación, con la finalidad de mejorar el sistema de selección de centros colaboradores con las prácticas externas en Educación Superior.

En este sentido, los objetivos específicos planteados en el estudio pretenden:

- Identificar los elementos clave para asegurar la calidad en la selección de los centros de prácticas.

- Diseñar una herramienta que nos ayude a valorar la idoneidad de los centros de prácticas como receptores de alumnado del Grado en Pedagogía.

- Aportar criterios para mejorar la selección de centros para el Prácticum que mejor se adecúen al perfil profesional del pedagogo, a partir de las valoraciones de sus agentes principales: los alumnos.

- Valorar las posibilidades de aprendizaje para los alumnos y el esfuerzo exigido por parte de los centros en prácticas.

- Comprobar el nivel de implicación por parte de los tutores profesionales en la formación del estudiante durante el periodo de prácticas.

- Comprobar el grado de implicación de los estudiantes en el centro de prácticas y la autonomía manifiesta a la hora de intervenir.

\subsection{METODOLOGÍA}

Esta investigación se sitúa en un enfoque mixto, de corte cuantitativo y cualitativo, con predominio este último. Analizar los datos mediante una metodología de este carácter nos ha permitido concretar de forma más minuciosa elementos claves y útiles para la selección de centros idóneos para la realización del Prácticum.

Para ello hemos empleado preguntas de respuesta abierta, lo que supone un mayor esfuerzo de transcripción de los datos, a lo que hay que añadir un cierto grado de subjetividad. Este coste de tiempo solamente es justificable si se trata, como es este caso, de resultados significativamente distintos a los extraídos de un cuestionario de preguntas cerradas para un análisis cuantitativo (Álvarez, 2003).

Este procedimiento metodológico se puede resumir en tres pasos:

- Establecer diferencias en los centros que pertenecen a distintos ámbitos de trabajo: educativo, social y de formación y gestión.

- Analizar las valoraciones del alumnado para la identificación de los factores que caracterizan a los centros de buenas prácticas.

- Realizar una propuesta de recomendaciones para la mejora de los procesos de formación inicial en su dimensión práctica que promuevan competencias de calidad. 


\subsection{POBLACIÓN Y MUESTRA}

La población de interés de nuestra investigación, tomando como criterio los objetivos planteados y de acuerdo con los datos facilitados por la Secretaría de la Facultad, ha quedado definida por el alumnado que durante el curso 2014-2015 ha cursado los 24 créditos de la asignatura Prácticum del Grado de Pedagogía de la Facultad de Educación de la Universidad de Salamanca. La muestra final está compuesta por 56 estudiantes del Grado en Pedagogía, de un total de 62, lo que supone un $90 \%$ de la población de referencia, e informa suficientemente de su representatividad, con edades comprendidas entre los 21 y los 38 años. Todos ellos respondieron voluntariamente al cuestionario elaborado para la valoración del centro en el que habían realizado sus prácticas externas una vez finalizado el proceso de evaluación del alumno, buscando así eliminar el sesgo que supone la influencia de la nota ante las respuestas elaboradas.

La muestra de centros donde los alumnos durante el curso 20142015 realizaron las prácticas externas quedó constituida en 48 centros, 22 de ellos relacionados con la orientación educativa (45.8\%), 6 con el ámbito de la Formación y Gestión (12.5\%) y 20 de ellos del ámbito social (41.6\%).

\subsection{INSTRUMENTO}

Para la recogida de la información se diseñó un instrumento ad hoc, que fue administrado en las aulas y que consta de cinco preguntas abiertas sobre las posibilidades de aprendizaje que ofrece el centro, el nivel de implicación por parte del tutor profesional, el grado de autonomía a la hora de intervenir, el esfuerzo exigido por parte del centro o persona responsable y, finalmente, una autovaloración del nivel de implicación por parte del alumno. Además, se han incluido otras 5 preguntas cerradas con una escala tipo Likert en las que se preguntan sobre los mismos aspectos con una valoración de tipo cuantitativo de 1-10.

Para la elaboración del instrumento se ha contado con un grupo de discusión en el que ha participado el profesorado perteneciente a la Comisión de prácticas externas de dicha titulación. Durante este proceso de elaboración del instrumento, se han identificado algunas dimensiones clave para la selección de los centros de prácticas, además de clasificar estas dimensiones en función del tipo de contribución que aportan al proceso de aprendizaje en el Prácticum. En este sentido, de acuerdo con Álvarez (2003, p. 47), estas preguntas de respuesta abierta nos van a permitir "fijar su propio nivel jerárquico de detalle de las modalidades o establecer el nivel de decisión en función de su experiencia”.

Este cuestionario ha permitido identificar los elementos clave para asegurar la calidad de los centros de prácticas que van a formar a los futuros pedagogos. Dicho instrumento incorpora cinco dimensiones persiguiendo, asimismo, encontrar unos criterios de calidad para la selección de centros excelentes, así como preservar, hasta donde sea posible, la objetividad en sus valoraciones, independientemente de las personas que asuman la responsabilidad de tutorizar este proceso en cada momento. Pretende, también, ser una herramienta útil para la toma de decisiones relativas a los cambios de centro de prácticas solicitados por el alumnado. Cuando un alumno presenta dificultades en sus prácticas, en ocasiones, es conveniente estudiar la posibilidad de abandonar el centro y buscar otro en el que pueda desarrollar su labor profesional satisfactoriamente. Esto es posible siempre y cuando se conozcan bien las características del centro correspondiente. 
Atendiendo a las distintas respuestas abiertas, se ha realizado una descomposición de cada dimensión, desglosándose en diferentes y numerosos factores. Por ello, una vez hecho el primer tratamiento de la información textual, se agrupan de la siguiente manera:

Tabla 1. Dimensiones y factores extraídos del análisis de contenido de las respuestas de los alumnos

\begin{tabular}{|c|c|}
\hline DIMENSIONES & FACTORES \\
\hline \multirow{10}{*}{ Posibilidades de aprendizaje } & Trabajo en equipo \\
\hline & Intervención directa \\
\hline & Organización institucional \\
\hline & Seguimiento \\
\hline & Presencia del Pedagogo \\
\hline & Colectivo \\
\hline & Diagnóstico \\
\hline & Diversidad de tareas \\
\hline & Relación teoría práctica \\
\hline & Aprendizaje autónomo \\
\hline \multirow{9}{*}{ Implicación del tutor profesional } & Constante \\
\hline & Trasparencia \\
\hline & Buena comunicación \\
\hline & Enseñanza \\
\hline & Resuelve dudas \\
\hline & Trabajo en equipo \\
\hline & Buen trato \\
\hline & Pautas \\
\hline & Autonomía \\
\hline \multirow{5}{*}{ Grado autonomía para intervenir } & Participación \\
\hline & Responsabilidad \\
\hline & Supervisión \\
\hline & Propuestas \\
\hline & Interés \\
\hline \multirow{4}{*}{ Esfuerzo exigido por el centro } & Adecuado \\
\hline & Elevado \\
\hline & Escaso \\
\hline & Autónomo \\
\hline \multirow{7}{*}{ Nivel de implicación alumnado } & Alta \\
\hline & Ambiente \\
\hline & Bajo \\
\hline & Tareas y Exigencias \\
\hline & Colectivo \\
\hline & Motivación \\
\hline & Autonomía \\
\hline
\end{tabular}


A partir de esta clasificación de las respuestas aportadas por los alumnos protagonistas del aprendizaje, en las diferentes categorías pueden reconocerse algunos de los elementos definitorios de un centro que permiten considerarlo adecuado para el desempeño de las prácticas de los futuros pedagogos.

\subsection{ANÁLISIS DE LOS DATOS}

Tras la cumplimentación del cuestionario por los estudiantes participantes, se ha llevado a cabo el análisis de datos. Para la parte cuantitativa se ha utilizado el paquete estadístico SPSS v. 21, y para la parte cualitativa se ha empleado el programa Gephi 8.0, el cual ha facilitado el tratamiento y la síntesis de la información textual recogida en la encuesta, así como el establecimiento de numerosos factores, obteniendo más del $90 \%$ de la información de tipo textual de las respuestas de los encuestados.

Este tratamiento de datos nos ha permitido centrarnos en dos cuestiones, por un lado, analizar y describir las respuestas, que los alumnos que acaban de realizar las prácticas externas en su ámbito profesional, dan a unas preguntas abiertas —a través de análisis de textos-y, por otro, ver en qué medida estas respuestas se relacionan — por el método de las especificidades - con algunas características propias de los tipos de centros en los que desarrollan tal labor profesional, y con las respuestas cerradas incluidas en el cuestionario.

Siguiendo algunas de las fases propuestas por Sánchez y Muñoz (2011), establecemos dos fases de análisis: en primer lugar, el estudio de textos, fase en la que creamos un glosario de los términos más utilizados y destacamos los contenidos y conceptos más relevantes y más utilizados por los alumnos, determinando así cada una de las dimensiones; y, en segundo lugar, la fase de relación de los datos obtenidos.

\section{RESULTADOS}

Desde un punto de vista cuantitativo, observamos en el Gráfico 1 las preguntas planteadas al alumnado y la valoración media obtenida en función del tipo de centro en el que han realizado sus prácticas. De forma general, se observa que aquellos alumnos que han realizado sus prácticas en un espacio enfocado a la formación y gestión educativa son los que valoran más positivamente todas las categorías a tener en cuenta, y por lo tanto son quienes manifiestan haber tenido menos problemas. Por el contrario, los alumnos que han realizado sus prácticas en un centro del ámbito social valoran con puntuaciones más bajas aspectos como las posibilidades de aprendizaje o el esfuerzo que se requiere a lo largo de las prácticas, y manifiestan que el grado de autonomía en estos centros es alto y la implicación por parte del alumno también. 
Gráfico 1. Valoración de los estudiantes ante los criterios de evaluación de los centros en prácticas

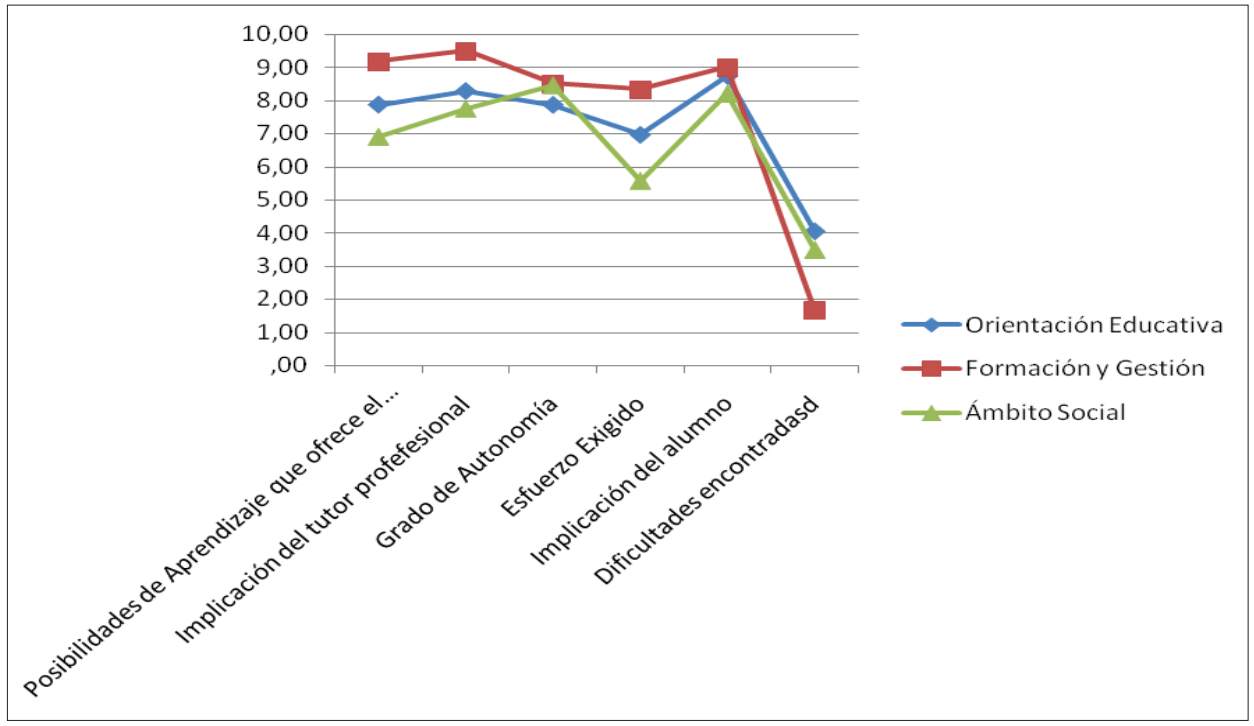

\subsection{POSIBILIDADES DE APRENDIZAJE DE LOS CENTROS DE PRÁCTICAS}

Centrándonos en la primera cuestión planteada a los alumnos, posibilidades de aprendizaje en los centros colaboradores en las prácticas del Grado en Pedagogía, son varias las categorías elaboradas para explicar esta cuestión, pudiendo visualizarse en la Figura 1 aquellos nodos cuyo valor asignado es mayor que el resto, así como las aristas de más grosor que son las conexiones entre los distintos nodos existentes.

Figura 1. Posibilidades de aprendizaje de los centros de prácticas colaboradores del Grado en Pedagogía

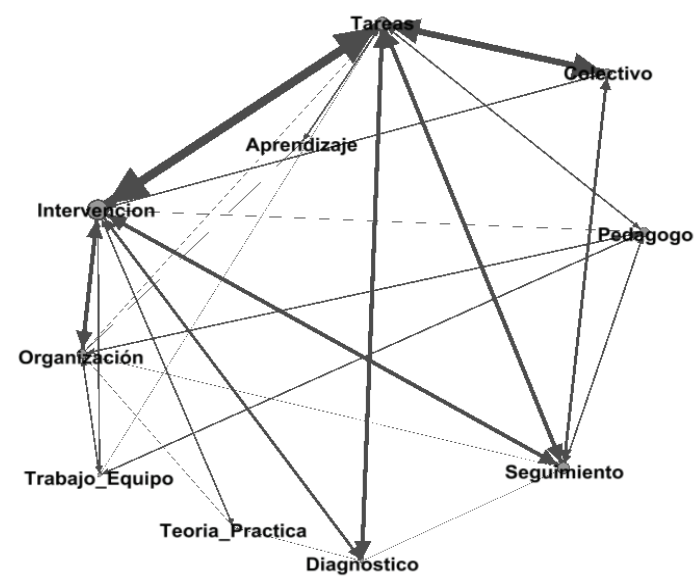


Los alumnos consideran que un centro ofrece posibilidades de aprendizaje cuando les da la posibilidad de realizar una intervención directa con los usuarios de los centros (impartición de clases, evaluar, implicarse en la resolución de conflictos, realizar talleres formativos, etc.), unida a la posibilidad de realizar una gran diversidad de tareas (diagnóstico, intervención, docencia, tareas administrativas, etc.), conociendo así el trabajo diario real del pedagogo. Sujeto 18: "He podido observar, intervenir, hacer programaciones y evaluaciones. Con lo cual creo que lo que he visto en mis años académicos he podido verlo en la realidad e implicarme en ellos" (Centro Educativo).

Por otra parte, los alumnos valoran positivamente obtener un seguimiento por parte del tutor profesional, donde sientan que se implica y explica claramente cuáles son sus tareas, cuáles van a ser las pautas de aprendizaje y las expectativas que el tutor tiene del alumno.

Las relaciones más intensas que se desprenden se encuentran entre las categorías diversidad de tareas-colectivo (oportunidad de conocer diferentes realidades de trabajo con colectivos en situación de riesgo, desventaja social, personas con discapacidad, minorías étnicas), aspecto señalado principalmente por los alumnos que se encontraban realizando sus prácticas en centros del ámbito social.

También se aprecia una relación intensa entre los nodos intervención-tareas, relación que tiene sentido en tanto la diversidad de tareas va a permitir al alumnado participar activamente en la acción.

Un aspecto relevante a destacar es la categoría presencia del pedagogo, el alumnado que ha realizado las prácticas en centros de ámbito social señalan, desde un punto de vista negativo, la falta de presencia del perfil del pedagogo en los centros, aspecto que les condiciona, apreciándose en las relaciones existentes entre variables, a la hora de realizar la diversidad de tareas, intervenir, conocer la organización y funcionamiento de los centros y aprender a trabajar en equipo. Sujeto 21: "Al no existir la figura del pedagogo, los aprendizajes son de tipo psicológico y también aprendizajes generales básicos en el trabajo de cualquier profesional. Los aprendizajes relacionados con la Pedagogía son limitados" (Gabinete Psicopedagógico).

\subsection{IMPLICACIÓN DE LOS TUTORES PROFESIONALES}

Si nos adentramos en la segunda cuestión, la implicación de los tutores profesionales en el proceso de aprendizaje de los alumnos en prácticas, estos valoran que los tutores se impliquen durante su periodo formativo de acuerdo a los siguientes criterios más destacados, que pueden visualizarse en la Figura 2. 
Figura 2. Implicación de los tutores profesionales en el proceso de aprendizaje de los alumnos en prácticas

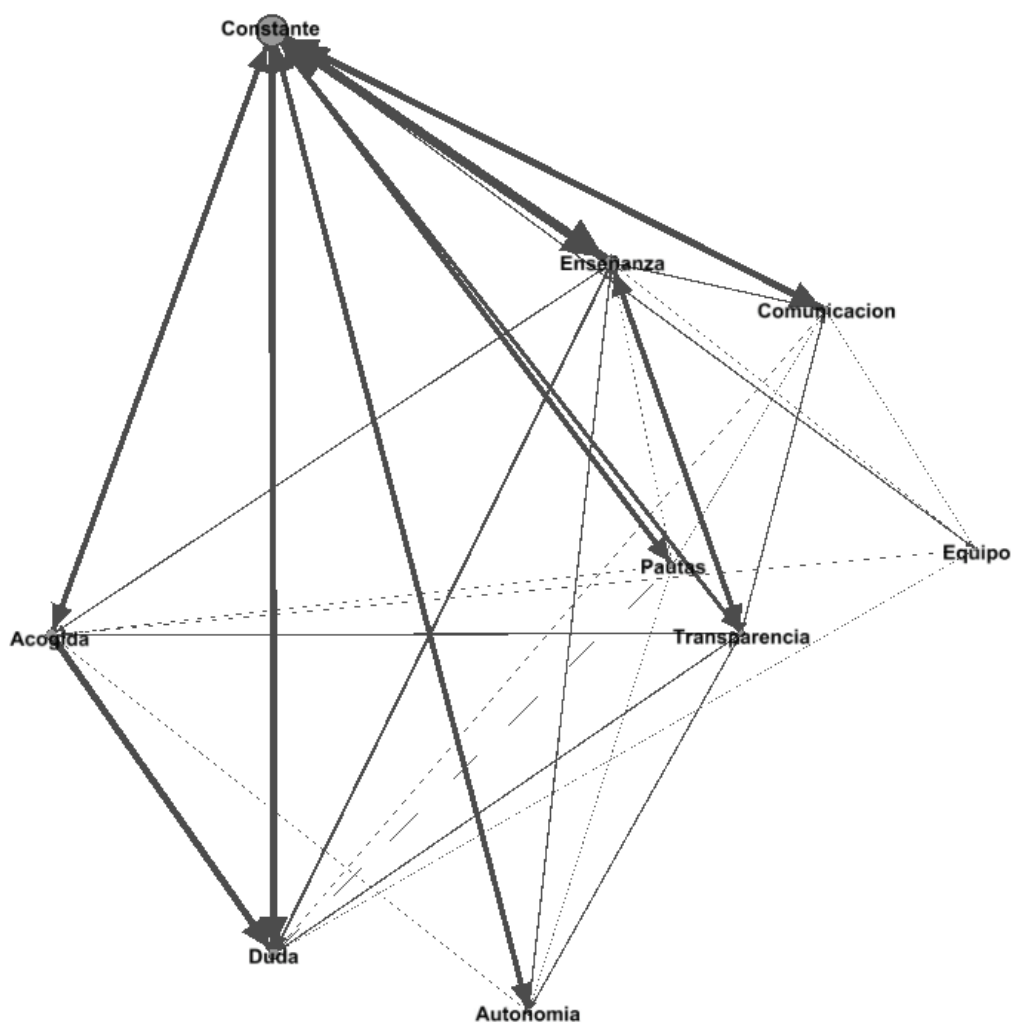

El primero de ellos alude al ser constante, siendo significativo e importante para el alumno que el tutor se encuentre presente en el centro durante el tiempo de prácticas, que mantenga un contacto directo y continuo y que ofrezca orientaciones a lo largo de este periodo. Los alumnos que destacan este aspecto en sentido negativo se refieren a que el tutor tiene falta de predisposición, normalmente por la cantidad de trabajo, especialmente señalado por alumnos que han realizado sus prácticas en centros de ámbito social, o en gabinetes privados.

Otro aspecto que define la implicación del tutor es la capacidad de enseñanza, en el sentido que el tutor sea capaz de transmitir conocimientos teóricos necesarios para comprender las intervenciones y el trabajo que debe realizar el alumno. También que exponga al alumno el plan de trabajo y debata y consensúe con él la adecuación del mismo, que muestre preocupación por su evolución en el proceso de aprendizaje y le ofrezca la posibilidad de realizar múltiples tareas, aspecto que en la cuestión anterior definía las posibilidades de aprendizaje de un centro en prácticas. Sujeto 15: "Su implicación desde el primer día ha sido excelente, ha preparado un plan de formación para mí que iba desde la base teórica hasta la autonomía práctica" (Centro educativo). 
Además de todo ello, los alumnos consideran que un tutor se implica si se ofrece a resolver las dudas que se le presentan al alumno, aspecto señalado principalmente por alumnos que han realizado sus prácticas en un centro de ámbito social o centro educativo. Los estudiantes destacan también la acogida por parte del tutor al inicio de sus prácticas, el trato agradable y la capacidad de hacer sentir cómodo al alumno durante este periodo formativo, así como la preocupación que muestra por conocerle y por integrarle en la institución.

Es reseñable que todas las categorías abordadas tienen relación, en mayor o menor intensidad, con el nodo constante, por lo que deducimos que el aspecto más importante para el alumnado en cuanto a la implicación del tutor se encuentra en la presencia y la asiduidad durante el periodo de aprendizaje práctico para el alumno, y que, por tanto, va a condicionar el resto de variables que ellos destacan como importantes. Principalmente, la relación entre constanteenseñanza es intensa, por lo que entendemos que los alumnos creen aprender más cuanto más contacto tienen con su tutor profesional. Al igual que ocurre con las categorías constante-dudas y constante-pautas, en este sentido la relevancia e importancia de la implicación del tutor estriba en estos condicionantes. Se desprende, por tanto, que el alumno siente la necesidad de mantener un contacto directo y diario con el tutor profesional, considerando que, de esa manera, le ofrecerá mayores pautas y resolverá sus dudas de forma más adecuada respecto a situaciones en las que este, en determinados momentos, se encuentra ausente.

Es importante señalar la relación establecida entre constante-comunicación y constante-transparencia, puesto que, por un lado, el alumno cree que es esencial mostrar una buena comunicación y que el tutor le manifieste, por diversos medios (teléfono, email, etc.), la preocupación por su bienestar durante el periodo de prácticas como signo de constancia y seguimiento. Por otra parte, es importante para el alumno la transparencia manifestada, puesto que para los estudiantes es necesario que el tutor les permita participar en las actividades de forma directa y que comparta toda la información relativa a los casos en los que trabaja, depositando en ellos, de esta forma, la confianza y la delegación de responsabilidades.

\subsection{GRADO DE AUTONOMÍA DEL ALUMNO EN EL CENTRO DE PRÁCTICAS}

Las respuestas relativas a la tercera cuestión planteada, grado de autonomía que posee el alumno a la hora de intervenir o actuar en el centro de prácticas, tienen una doble orientación, positiva y negativa en función de la tipología de centro donde se han realizado las prácticas. En este sentido, los alumnos que han realizado sus prácticas en una institución de ámbito social realizan una valoración media alta de este aspecto, pero eso no significa que sea una valoración positiva, puesto que la autonomía conlleva una doble connotación, por un lado, alude a la capacidad de iniciativa por parte del alumno en la intervención, por otro lado hace referencia a las situaciones en las que el alumno debe actuar de forma independiente por falta de directrices del tutor de prácticas. 
Figura 3. Grado de autonomía del alumno en el centro de prácticas

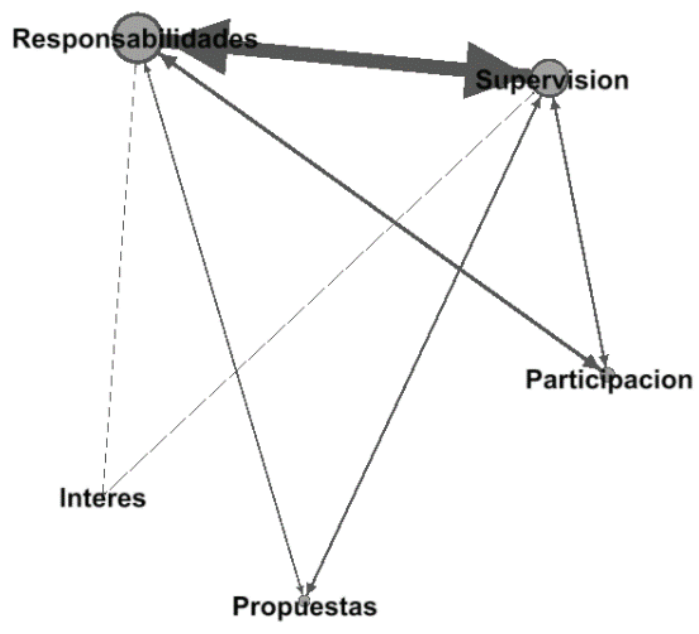

Se observa en la Figura 3 que son dos las categorías que principalmente definen la autonomía del alumno respecto a la institución que les acoge, de una parte la responsabilidad, de tal forma que se permite al alumno actuar de forma independiente previa observación. El tutor ofrece al alumno intervenir en aquellas actividades que considera formativas y le permiten hacerse cargo y responsable de algunas tareas. Por otro lado, se admite que el alumno ponga en práctica actividades propuestas y diseñadas a partir de los conocimientos teóricos adquiridos. Sujeto 42: "El tutor profesional me dio unas pautas al principio de las prácticas, pero en todo momento mi grado de autonomía ha sido alto. Me dejaban intervenir, aportar ideas, estrategias, etc." (Centro de atención a personas de etnia Gitana).

La categoría relativa a la responsabilidad es valorada negativamente, principalmente por alumnos que han realizado las prácticas en gabinetes de ámbito privado y determinados centros de ámbito social, puesto que el estudiante manifiesta que le permiten hacerse responsable de algunas actuaciones sin supervisión por parte del tutor, aspecto relacionado con la siguiente categoría que más frecuencia de respuestas obtiene y con la que se establece una relación intensa, como es la supervisión.

El alumno manifiesta que su autonomía en el centro de prácticas debe ir acompañada de una supervisión por parte del tutor profesional, de tal forma que le ofrezca las orientaciones, pautas y directrices necesarias, le explique la forma en la que debe intervenir en cada caso, resuelva las dudas surgidas antes de actuar, supervise las tareas realizadas y ofrezca la posibilidad de ponerlo en común con otros compañeros. Quienes destacan este aspecto de forma negativa, principalmente son los que han realizado sus prácticas en una institución de ámbito social, destacando la falta de directrices y supervisión por parte del tutor para saber si las tareas se realizaban correctamente, así como la ausencia del tutor a la hora de actuar. Sujeto 37: "Teníamos demasiada autonomía, tanta que interveníamos sin la supervisión de alguien que nos dijese si lo que hacíamos estaba bien o mal, por lo que el aprendizaje no puede ser satisfactorio" (Centro de atención a personas con discapacidad). 


\subsection{NIVEL DE ESFUERZO EXIGIDO AL ALUMNO}

La cuarta pregunta planteada pretendía descubrir el esfuerzo exigido al alumno desde el centro de prácticas o por parte del tutor profesional. La mayoría de los estudiantes cree que el esfuerzo exigido ha sido el adecuado, en tanto el tutor adapta las exigencias del alumno y realiza una adecuada gradación en la realización de tareas que solicita al mismo y posteriormente la autonomía en la intervención de forma progresiva. La mayoría de alumnos cree que el esfuerzo es adaptado a una actividad de prácticas donde la función principal es aprender, ajustado a las horas de prácticas y a las capacidades del alumnado.

Figura 4. Esfuerzo exigido al alumno durante sus prácticas

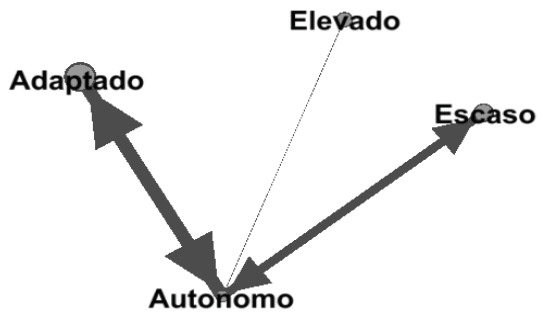

Quienes opinan que el esfuerzo ha sido escaso, argumentan que no se exige un mínimo de responsabilidad, que el tutor no demanda tareas concretas por considerar que se trata de una tarea formativa y un primer contacto con la realidad, sin embargo, en determinadas ocasiones la escasez de labores encomendadas está vinculada a la falta de tiempo del tutor profesional, principalmente en centros de ámbito social.

Los que creen que el esfuerzo es elevado, argumentan que la institución requiere presencia y dedicación en el centro, así como trabajo autónomo en casa. Consideran que es signo de esfuerzo exigido elevado, que el tutor profesional, una vez realizadas las tareas por parte del alumno y supervisadas, les exigen volver a realizarlas con el objetivo de seguir aprendiendo. Quienes destacan que el esfuerzo exigido es elevado en un sentido negativo, lo argumentan diciendo que la relación institución-alumno de prácticas no puede convertirse en una relación profesional y las exigencias no pueden ser las mismas que a un trabajador, puesto que para ellos es una experiencia dentro de un proceso de enseñanza-aprendizaje.

Nuevamente, se manifiesta en esta cuestión la categoría autonomía. Observamos en la Figura 4 que, aun teniendo menor valor que el resto, es importante en sí misma porque guarda relación con el resto de nodos. Determinados alumnos destacan que el esfuerzo exigido depende de la implicación que el alumno está dispuesto a ofrecer, argumentando que la institución y el tutor profesional, una vez superada la fase de observación, les permite actuar al ritmo que ellos mismos van marcando. El centro, una vez que el alumno cumple las exigencias mínimas, se adapta a él y le ofrece la posibilidad de implicarse en la medida que considere, dándole la oportunidad de realizar más o menos tareas en función de las propuestas realizadas, aspectos destacados por estudiantes que han estado en centros educativos y en algunos centros relacionados con la formación y gestión educativa. 


\subsection{NIVEL DE IMPLICACIÓN DEL ALUMNO EN EL CENTRO DE PRÁCTICAS}

Una vez cuestionados los estudiantes respecto a los temas relativos al centro y tutor profesional, era importante conocer la opinión que los alumnos manifiestan en torno a su nivel de implicación en el centro de prácticas. Como se observa en la Figura 5, tres son las categorías que mayor frecuencia de respuesta han obtenido.

Figura 5. Nivel de implicación de los alumnos en el centro de prácticas

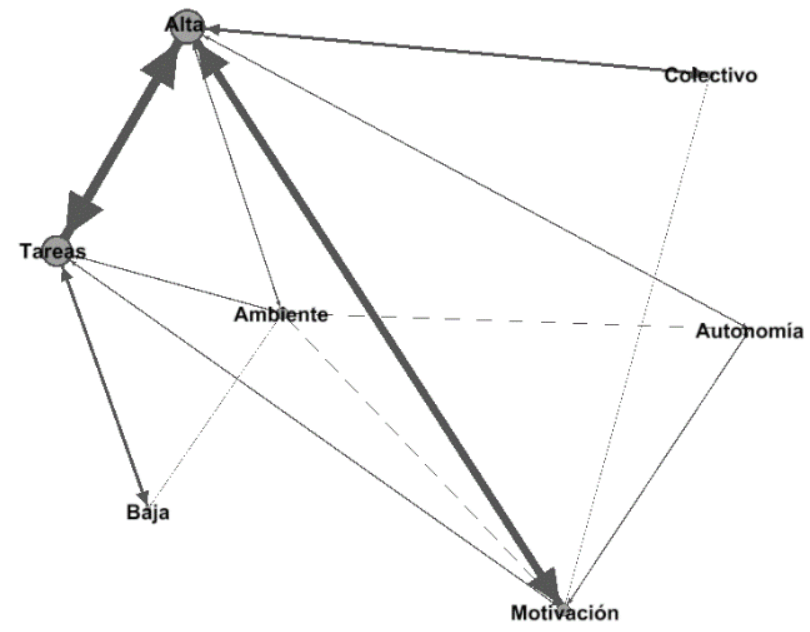

Los que creen que su implicación ha sido alta, argumentan que han cumplido con el trabajo exigido, se han implicado en conocer cómo trabaja su tutor y el resto de profesionales con los que se ha relacionado, han mostrado disposición ante cualquier iniciativa, trabajo o experiencia y han realizado propuestas y sugerencias de actividades no requeridas por el centro o tutor. Además, han realizado un trabajo de preparación en casa y más horas de las requeridas, argumentos similares a los ofrecidos para definir que el esfuerzo exigido en el centro es elevado, como hemos visto anteriormente. Como afirman los siguientes sujetos: Sujeto 6: "En todo momento he aceptado todas las tareas que me proponía la orientadora y, en algunos casos, con previa preparación en casa" (Centro educativo); Sujeto 31: "he realizado todas las tareas exigidas, me he interesado por aprender y conocer todo lo que el centro ofrecía, estaba muy motivada por aprender y siempre predispuesta" (Centro de protección de menores).

La implicación del alumno también aumenta cuando le solicitan la realización de tareas que le motivan y le gustan, e incrementa su interés por aprender y conocer todo lo que la institución les ofrece, manteniendo así una predisposición positiva al trabajo encomendado, aspectos destacados principalmente por estudiantes que han realizado sus prácticas en algún centro educativo o de ámbito social, concretamente aquellos que han trabajado con colectivos en desventaja o riesgo de exclusión social. En este sentido, creen 
que se implican cuando realizan las tareas encomendadas y requeridas cumpliendo así con las exigencias del tutor, incluidas aquellas que los estudiantes consideran que no son competencia y responsabilidad directa del pedagogo, cuando siguen en contacto con la institución finalizado el periodo de prácticas.

Unido a lo anterior, otra de las categorías que obtiene mayor valor y que se relaciona intensamente con la implicación del alumno es la motivación, ya sea personal por el colectivo con el que va a trabajar, por ejemplo, o profesional por conocer el funcionamiento de la institución y funciones del pedagogo.

Las relaciones que se establecen de manera más intensa se encuentran entre las categorías descritas, motivación-implicación alta y tareas-implicación alta. Es algo intrínseco a la asignatura Prácticas Externas que el alumno se sienta motivado por este periodo, pues es su único contacto con la realidad profesional (Casillas et al., 2015). No obstante, mantener esa motivación a lo largo de los meses de prácticas es un hecho que va a condicionar la implicación de los alumnos, como también el tipo de trabajo encomendado por la institución, que no siempre es el mismo en función del ámbito de destino y tutor profesional.

\subsection{DIFICULTADES ENCONTRADAS POR EL ALUMNADO DURANTE EL PERIODO DE PRÁCTICAS}

Como se ha observado en la Figura 1, los alumnos, en general, no consideran haber tenido dificultades a lo largo del periodo de prácticas. Aun así, supone un problema enfrentarse al periodo de prácticas si no existe en la institución la figura del pedagogo, aunque el tutor y otros profesionales estén vinculados con el ámbito de la pedagogía. Es también una dificultad añadida tener discrepancias con el tutor profesional respecto a los objetivos que se deben cumplir a lo largo de este periodo, la falta de intercambio de información por parte del responsable de aspectos relativos a su trabajo diario, o la falta de contacto presencial con el tutor que debe cumplir con múltiples obligaciones que no le permiten ofrecer una adecuada tutorización al alumno. Sujeto 29: "todas las dificultades con las que me he podido encontrar han sido debidas a la inexistencia de un buen tutor de prácticas" (Centro de atención a personas con discapacidad). Sujeto 52: "al no existir la figura del pedagogo, en ocasiones me sentí un poco desplazada profesionalmente" (Centro hospitalario).

Con igual frecuencia de respuesta se establece la categoría intervención, que alude a dos situaciones, por una parte, hay alumnos que resaltan como dificultad haber intervenido poco en trabajo de campo, y por otra, señalan haber tenido problemas de adaptación al colectivo y al tipo de centro, encontrando dificultades a la hora de intervenir ante situaciones conflictivas o derivadas de conductas inadecuadas por parte de los usuarios. 
Figura 6. Dificultades encontradas a lo largo del periodo de prácticas

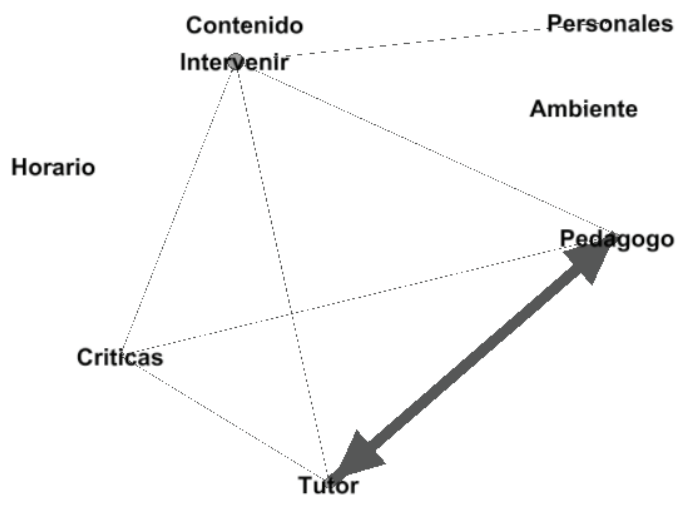

La relación más intensa, tal como se observa en la Figura 6, se desprende de los nodos pedagogo y tutor. Esta dificultad, en sí misma, puede suponer para el alumno una posibilidad de aprendizaje, en tanto debe ser capaz de mostrar las habilidades y competencias profesionales adquiridas a lo largo del Grado en cuanto a las relaciones profesionales que va a establecer en un futuro próximo. Saber enfrentarse a las dificultades de relación con otros compañeros y resolver conflictos de convivencia es fundamental para una adecuada integración en determinados equipos interdisciplinares de trabajo. No obstante, no se debe olvidar que esta relación no se establece en términos de igualdad, pues el alumno es conocedor de que el tutor profesional va a realizar una evaluación (del mismo) y esto puede condicionar las maniobras que el estudiante pueda realizar en torno a la resolución de conflictos que puedan surgir. Es fundamental, por otra parte, incidir en que los centros colaboradores de prácticas cuenten con la figura del pedagogo, hecho que no siempre es posible, porque determinados puestos laborales requieren perfiles de acceso diverso, encontrando de esta forma que el tutor es psicólogo, educador o maestro.

\section{CONCLUSIONES, DISCUSIÓN Y PROPUESTAS}

A partir de los resultados obtenidos, extraemos las principales características que debe poseer una institución para colaborar con las prácticas externas y, por lo tanto, los centros serán más adecuados para proporcionar una formación práctica al alumnado universitario.

Partimos de la idea de que un centro de prácticas idóneo debe ofrecer al alumnado posibilidades reales de aprendizaje, debe contar con un tutor profesional implicado en la formación práctica del estudiante, debe ofrecer una cierta autonomía al alumno universitario que, al encontrarse en último curso, ya dispone de suficiente formación teórica para poder realizar diversas tareas propias de su profesión. A partir de los resultados obtenidos, se concluye que los centros en prácticas deben prestar al alumno la posibilidad de realizar todas, o casi la totalidad de las funciones que un pedagogo desempeña en su puesto de trabajo. Sea cual sea la institución, sería deseable que ofrecieran la oportunidad de realizar intervenciones directas con los alumnos, familias, profesores o usuarios de la institución, y proporcionar 
al alumno un seguimiento durante el periodo de prácticas; aspectos todos que definen y diferencian un centro que ofrece unas claras posibilidades de aprendizaje al alumnado.

En segundo lugar, la institución de prácticas debe proporcionar un tutor profesional comprometido y capacitado para cumplir con las funciones encomendadas. Se requieren profesionales constantes, con amplia capacidad de enseñanza y de resolución de dudas teórico-prácticas durante el periodo de formación. Fundamental es que sea pedagogo de formación, o con titulaciones próximas y cercanas al ámbito pedagógico. Molina (2007) señala que es relevante, para mejorar los resultados, establecer un punto de encuentro entre los tutores de la Universidad y los tutores del centro, que a menudo viene determinado por establecer una fuerte coordinación entre ambos, estrechando la relación y realizando más reuniones. Sin embargo, estas relaciones, como señala Pérez (2005), no han estado exentas de polémicas, así como de gran desconfianza y falsas creencias entre estas dos figuras tan importantes en las prácticas.

En tercer lugar, los alumnos sienten la necesidad de obtener de las instituciones prácticas cierto grado de autonomía a la hora de desenvolverse tanto en el centro como en el planteamiento de actividades o tareas que repercutan en un mayor aprendizaje. Esta autonomía debe basarse en la característica esencial de la responsabilidad, ya que los estudiantes necesitan sentirse responsables de su trabajo en el centro de prácticas, aunque requieran supervisión constante por parte del tutor profesional.

Es sorprendente la necesidad que el alumno tiene de una autonomía vigilada, sentirse libres pero seguros y respaldados, y es esencial plantear los porqués de esta cuestión, así como el hecho de que el estudiante no sea capaz de valorar, desde un punto de vista profesional, si las actuaciones que ha llevado a cabo de forma autónoma han tenido una repercusión positiva en los sujetos (objeto de la misma), y necesiten una valoración que se traduzca en evaluación por parte del tutor.

Este hecho puede ser significativo de una inseguridad estudiantil que quizá es necesario mitigar desde las aulas trabajando a partir de sólidos criterios que fomenten competencias profesionales basadas en la responsabilidad y autonomía de los estudiantes, que permitan que en el momento de las prácticas externas el alumno salga temporalmente del rol de estudiante y sea capaz de poner en práctica sus competencias profesionales. Formar a alumnos dependientes de la valoración constante y continua del profesor y del tutor no fomenta la independencia, la capacidad de innovación o resolución de conflictos y esto, en determinados momentos y con determinados centros e instituciones, puede ocasionar problemas a lo largo del periodo de prácticas. Es esta una cuestión en la que debería centrarse la Universidad: fomentar la autonomía en tanto que el alumno sea capaz de realizar las tareas encomendadas y, además, realizar propuestas a las instituciones donde realiza la práctica que le ayuden a formarse y hacerse valer como profesional.

En otro orden de cosas, consideramos que para que la relación establecida entre el centro, el tutor profesional de prácticas y el alumno funcione, deben cumplirse unas mínimas exigencias de esfuerzo e implicación por parte del alumnado. A partir de los datos obtenidos y examinados se observa que los centros de prácticas y los tutores profesionales, en general, adecúan correctamente el nivel de esfuerzo exigido a los estudiantes. No obstante, hay un amplio grupo de alumnos que consideran que el esfuerzo exigido es elevado. Desde nuestra posición, podemos cuestionar si los argumentos aportados por los estudiantes (trabajo autónomo en casas, presencia y dedicación en el centro, alguna hora más de las estipuladas para prácticas, solicitud de repetición de actividades o tareas que 
permitan seguir aprendiendo al alumno) son signo de un elevado esfuerzo, puesto que la presencia en el centro y el trabajo autónomo son considerados mínimos exigidos para la superación de la asignatura Prácticum, en tanto deben realizar en paralelo la memoria exigida desde la Facultad.

También es fundamental que los estudiantes encuentren fuentes de motivación vinculadas a las instituciones o tutores profesionales que fomenten una adecuada implicación del alumno por aprender, por colaborar, por desempeñar las tareas y funciones encomendadas, etc. No siempre es fácil mantener cotas de motivación elevadas, puesto que el Prácticum implica que los alumnos se envuelvan en un entorno laboral que en la mayoría de las ocasiones es desconocido para ellos, influyendo, igualmente, la percepción previa que tenían del centro en prácticas y la realidad en que se encuentran, la idea del trabajo profesional del pedagogo y las funciones que en su día a día realiza en función de la institución de referencia, etc.

Para finalizar de elaborar el mapa de criterios que nos ayuden a discernir entre centros idóneos para dirigir a los alumnos en prácticas del Grado en Pedagogía, debemos, en la medida de las posibilidades, evitar situaciones que puedan ocasionar problemas a los estudiantes, a los propios centros o incluso a la Universidad. Como hemos mencionado anteriormente, es fundamental contar con figuras profesionales que cumplan las funciones de tutor de alumnos y que estos sean pedagogos, puesto que se ha comprobado que los mayores conflictos están ocasionados por discrepancias entre los alumnos y aquellos tutores profesionales no vinculados directamente con el ámbito de la pedagogía, coincidiendo, algunos de ellos, con los citados en las investigaciones sobre el tema. En la literatura encontramos autores como Zabalza (2004), Pérez (2011), Vila et al. (2012) y Rodríguez (2011) que realizan una interesante revisión sobre estos criterios que sirven para analizar la calidad del Prácticum.

En la siguiente representación (Figura 7) se recogen los criterios esenciales a los que hemos llegado tras la realización del estudio, para la elección de centros de prácticas de calidad.

Figura 7. Criterios para la selección de centros de prácticas de calidad

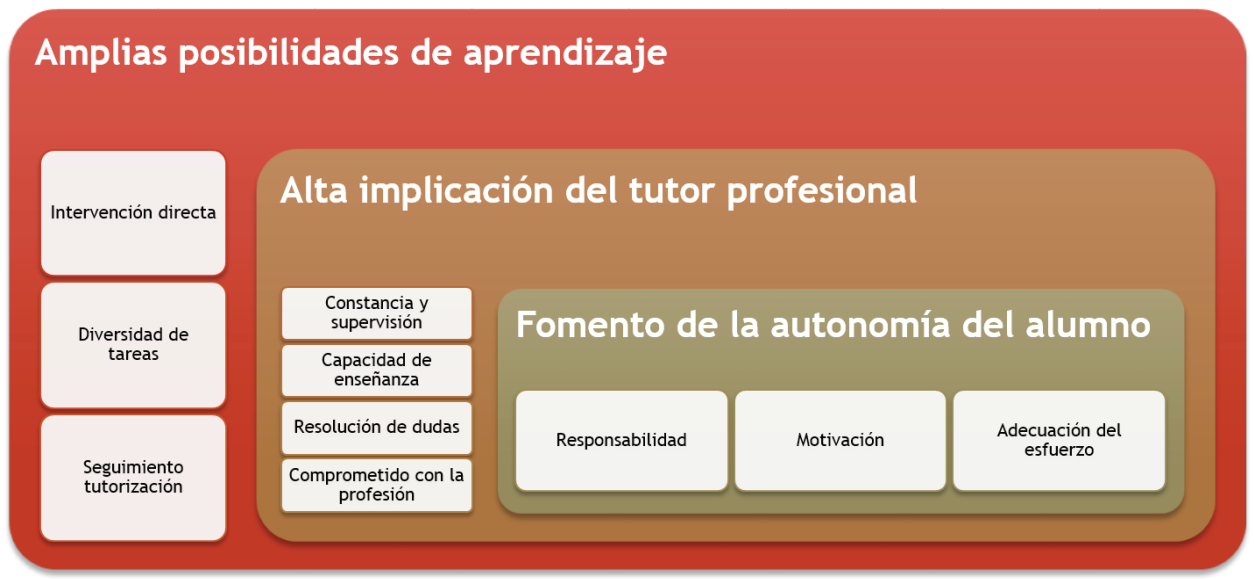


Estudios Pedagógicos XLII, N 3: 369-389, 2016

ESTUDIO DE LOS CRITERIOS PARA LA SELECCIÓN DE CENTROS DE PRÁCTICAS DE CALIDAD. UNA PROPUESTA

DE EVALUACIÓN PARA MEJORAR LA FORMACIÓN DE LOS PEDAGOGOS

\section{REFERENCIAS BIBLIOGRÁFICAS}

Álvarez, R. (2003). Las preguntas de respuesta abierta y cerrada en los cuestionarios. Análisis estadístico de la información. Metodología de Encuestas, 5(1), 45-54.

Armengol, C., Castro, D., Jariot, M., Massot, M., \& Sala, J. (2011). El Practicum en el Espacio Europeo de Educación Superior (EEES): mapa de competencias del profesional de la educación. Revista de Educación, 354, 71-98.

Bas, E. (2005). El Practicum en las titulaciones de Pedagogía y Educación Social: entre el discurso y sus prácticas. Education, 23, 191-206.

Bolívar, A., \& Domingo, J. (2006). The professional identity of secondary school teachers in Spain: Crisis and reconstruction. Theory and Research in Education, 4(3), 339-355.

Casillas, S., González, M., \& Serrate, S. (2015). Percepción de los estudiantes del Grado en Pedagogía y Educación Social sobre la organización, utilidad y formación del Prácticum. Revista Enseñanza y Teaching, 33(2), 171-190. doi:10.14201/et2015332171190

Flores, M. A. (2006). Induction and mentoring. Policy and practice. In J. R. Dangel (Ed.), Research on Teacher Induction. Teacher Education Yearbook XIV (pp. 37-66). Toronto: Rowan and Littlefield Publishers.

Gairín, J., \& Martín, M. (2004). Las instituciones educativas en la encrucijada de los nuevos tiempos: retos, necesidades, principios y actuaciones. Tendencias Pedagógicas, 9, 21-44. Recuperado de http://goo.gl/GW97hz

González, M. (2009). Integración de práctica y reflexión en un único proceso. Revista Portuguesa de Pedagogía, 43(2), 23-40.

González, X.A., \& Hevia, I. (2011). El Practicum de la Licenciatura de Pedagogía: estudio empírico desde la perspectiva del alumnado. Revista de Educación, 354, 209-236. Recuperado de http:// goo.gl/GbRLal

López, M. C., \& Hinojosa, E. (2008). Percepciones iniciales de los estudiantes sobre la formación práctica. Revista Iberoamericana de Educación, 47(5), 1-12. Recuperado de http://goo.gl/ TAFHbA

Marcelo, C. (2009). Los comienzos en la docencia: un profesorado con buenos principios. Profesorado: revista de curriculum y formación del profesorado, 13(1), 1-25.

Molina, E. (2007). Coordinación de las actuaciones de Prácticum: Niveles y compromisos. IX Symposium Internacional sobre Practicum y Prácticas en empresas en la formación universitaria. Poio, Pontevedra.

Morales, S. (2010). El Practicum en educación social: el portafolio como instrumento de seguimiento y evaluación del aprendizaje de los alumnos. Revista Docencia e Investigación, 20, 33-58. Recuperado de http://goo.gl/s7isBi

Morales, S. (2013). Evaluando el practicum en Educación Social: acciones de mejora ante la puesta en práctica de los nuevos grados. Revista de Docencia Universitaria, 11(1), 349-364. Recuperado de http://www.red-u.net/

Pérez, M.P. (2005). ¿Se pueden determinar las funciones del supervisor universitario? Revista de Investigación Educativa, 23(2), 315-332.

Pérez, P.J. (2011). Prácticum de calidad para el Máster docente de Secundaria. Participación Educativa, 16, 114-121. Recuperado de http://goo.gl/Wk1n81

Rodríguez, S. (2011). La evaluación del practicum y los procesos de acreditación de titulaciones. En M. Raposo, M. E. Martínez, P. C. Muñoz, A. Pérez, \& J.C. Otero (Coords.), Evaluación y supervisión del practicum: el compromiso con la calidad de las prácticas (pp. 57-100). Santiago de Compostela: Andavira.

Sánchez, M., \& Muñoz, A. (2001). Análisis de respuestas abiertas en un cuestionario sobre conductas relacionadas con el SIDA. Revista de Psicología Social: International Journal of Social Psychology, 16(3), 261-273. 
Valle, J. M., \& Manso, J. (2011). La nueva formación inicial del profesorado de Educación Secundaria: modelo para la selección de buenos centros de prácticas. Revista de Educación, 354, 267-290.

Vilà, R., Aneas, A., Burguet, M., Millán, D., Noguera, E., \& Rajadell, N. (2012). Guia Pràctiques Externes del Grau de Pedagogia UB per a les organitzacions. Depósito digital de la UB. Recuperado de http://hdl.handle.net/2445/32365

Zabalza, M. A. (2004). Condiciones para el desarrollo del practicum. Profesorado. Revista de Currículum y Formación del Profesorado, 8(2). Recuperado de http://www.ugr.es/ recfpro/ rev82ART1.pdf

Zabalza, M. A. (2006). El Practicum y la formación del profesorado: balance y propuestas para las nuevas titulaciones. En J. M. Escudero (Coord.), La mejora de la educación y la formación del profesorado. Políticas y prácticas (pp.309-330). Barcelona: Octaedro.

Zabalza, M. A. (2011). El Practicum en la formación universitaria: estado de la cuestión. Revista de Educación, 354, 21-43. Recuperado de http://www.revistaeducacion.mec.es/re354/re354_02.pdf 
